\title{
Optimistic vs. pessimistic endings in climate change appeals
}

\author{
Brandi S. Morris (i] ${ }^{1 凶}$, Polymeros Chrysochou (D) ${ }^{1,2}$, Simon T. Karg ${ }^{1}$ \& Panagiotis Mitkidis ${ }^{1,3}$
}

The use of emotion in climate change appeals is a hotly debated topic. Warning about the perils of imminent mass extinction, climate change communicators are often accused of being unnecessarily 'doomsday' in their attempts to foster a sense of urgency and action among the public. Pessimistic messaging, the thinking goes, undermines engagement efforts, straining credulity and fostering a sense of helplessness, rather than concern. Widespread calls for more optimistic climate change messaging punctuate public discourse. This research puts these claims to the test, investigating how affective endings (optimistic vs. pessimistic vs. fatalistic) of climate change appeals impact individual risk perception and outcome efficacy (i.e., the sense that one's behavior matters). The findings of three online experiments presented in this paper suggest that climate change appeals with pessimistic affective endings increase risk perception (Studies 1 and 2) and outcome efficacy (Study 3), which is the result of heightened emotional arousal (Studies 1-3). Moreover, the results indicate that the mediating effect of emotional arousal is more prevalent among political moderates and conservatives, as well as those who hold either individualistic or hierarchical world views. Given that these audiences generally exhibit lower risk perception and outcome efficacy in relation to climate change, the results suggest that climate change appeals with pessimistic endings could trigger higher engagement with the issue than optimistic endings. These findings are interpreted in light of recent research findings, which suggest that differences in threat-reactivity and emotional arousal may be attributable to brain functions/anatomy mappable to basic motivations for safety and survival. Implications for scholars and practitioners are discussed.

\footnotetext{
${ }^{1}$ Department of Management, Aarhus University, Aarhus, Denmark. ${ }^{2}$ Ehrenberg-Bass Institute, UniSA Business, University of South Australia, Adelaide, Australia. ${ }^{3}$ Social Science Research Institute, Duke University, Duke, USA. ${ }^{\bowtie}$ email: bsm@mgmt.au.dk
} 
"I don't want your hope. I want you to panic. I want you to feel the fear I do. Every day. And want you to act. I want you to behave like our house is on fire. Because it is."

-Greta Thunberg (World Economic Forum's Annual Meeting in Davos, 2019)

\section{Introduction}

hould climate change appeals have optimistic or pessimistic endings? On the one hand, pessimistic messaging, often characterized as "climate disaster porn," may be as harmful to engagement efforts as outright denial (Mann et al., 2017) if it fosters paralysis rather than action (Freedman, 2017). On the other hand, though optimistic messaging may comfort a public suffering from chronic 'apocalypse fatigue' (Nordhaus and Shellenberger, 2009), it might not spark the affective engagement critical for triggering risk perception (Slovic et al., 2004).

Affect is a powerful lever that can cause us to be overly sensitive to small changes in the environment while distracting us from large shifts of much greater consequence (Slovic et al., 2004). It can prompt irrational levels of alarm regarding threats with low levels of likelihood (Rottenstreich and Hsee, 2001), and deluded levels of optimism in the face of potentially catastrophic consequences (Sharot, 2011). In the context of climate change, positive affect leads to avoidance of new information, which could potentially cause distress, whereas negative affect has the opposite effect (Yang and Kahlor, 2013). In risk management, negative affect is widely acknowledged as the "wellspring of action" (Peters and Slovic, 2000), and this has shown to be no less true for the threat of climate change (Schwartz and Loewenstein, 2017). Such evidence diverges from findings in the field of health communication, where positive affect encourages information-seeking (Schwarzer and Jerusalem, 1995; Yang et al., 2011). One plausible explanation for this inverse effect is perceived efficacy, an important predictor of engagement with climate change (Feldman and Hart, 2015; Kellstedt et al., 2008). In other words, people are less likely to take action when they feel overwhelmed or hopeless (Bandura, 2002; Lorenzoni et al., 2007; Mayer and Smith, 2019).

Negative affect has been shown to increase estimations of risk probability while positive affect reduces them (Finucane et al., 2000; Ganzach, 2000). In line with the principle of loss aversion (Tversky and Kahneman, 1992), negative affect is more likely to be associated with loss rather than gain frames. A number of studies show that sadness (Schwartz and Loewenstein, 2017), worry (Smith and Leiserowitz, 2014), fear (Feldman and Hart, 2015), anxiety (Weber, 2006), hope, and anger (Feldman and Hart, 2015) are strongly associated with climate change engagement, while others observe no association with anger or fear (Smith and Leiserowitz, 2014). Witte and Allen (2000) find that fear appeals are ineffective when perceived efficacy is low. Moreover, negative affect can be considered a form of cognitive discomfort, and as the peak end rule illustrates (Do et al., 2008), people are willing to choose situations with objectively more overall pain as long as the event ends with relatively less pain. For this reason, we focus on how valence at the end of a climate change appeal influences a receiver's risk perception of climate change-related consequences, and their perceived outcome efficacy.

The assessment of risk is subjective and inextricably linked to judgments made on the basis of core values; people tend to subconsciously avoid and mistrust information that threatens their identity or values, or which has the potential to cause estrangement from social in-groups (Kahan, 2015). Belief in anthropogenic climate change is associated with liberal ideology (McCright et al., 2016) because the acknowledgment that human activity is influencing the climate implies a need for regulation.
Beyond political beliefs, cultural cognition theory stipulates that core values shape information processing and risk assessment along two dimensions or cultural worldviews: 'group' and 'grid' (Kahan and Braman, 2006). Research suggests that group/grid cultural worldviews predict beliefs about climate change better than any other individual characteristic (Kahan et al., 2011). The 'group' dimension categorizes people as either 'individualists' or 'communitarians' based on their beliefs about how strongly people are bound to other members of society. The 'grid' dimension describes values about the degree to which an individual believes their choices are controlled and limited by their roles within society.

In this paper, we make two propositions. First, the affective ending (i.e., optimistic vs. pessimistic) of climate change appeals impacts people's risk perception and perceived outcome efficacy, which is mediated through emotional arousal. We suggest that climate change appeals with pessimistic endings positively influence climate change risk perception and outcome efficacy because they heighten emotional arousal. Second, the strength of the proposed mediated relationship is attenuated by the values of a message receiver. We predict a less pronounced effect for those with liberal ideology, including those holding communitarian or egalitarian worldviews, than for conservatives and those holding individualist or hierarchical worldviews.

Across three experiments, we provide support for our propositions. In Study 1, we test the mediating role of emotional arousal on the impact of a climate change text with an optimistic vs. pessimistic ending on risk perception. It is important to note that we do not equate negative valence with fatalism. The pessimistic ending still presents the possibility of turning things around. Further, we test the moderating role of political ideology. In Study 2, we successfully replicate Study 1 by using a video stimulus combined with text, increasing the ecological validity of our findings. We also test the moderating role of message receiver values by adding a more nuanced measure of ideological commitments: cultural worldviews (Kahan et al., 2009), in addition to political ideology. Finally, in Study 3, using a nationally representative sample of the U.S. population, we add a fatalistic condition and introduce outcome efficacy as a dependent measure.

\section{Study 1: The influence of affective ending (optimistic vs. pessimistic) on emotional arousal and perception of climate change risk-written stimuli}

Experimental design. In Study 1, participants were presented with a climate change text that had either an optimistic or pessimistic ending. In a single-factor (affective ending: optimistic $(N=101)$ vs. pessimistic $(N=99))$ between-subjects online experiment $(N=200$ U.S.-based residents were recruited through Mturk $\left(35.5 \%\right.$ females, $\left.M_{\text {age }}=34.6, \mathrm{SD}_{\text {age }}=10.4\right)$ for a compensation of USD 1.20. Sample size was determined beforehand (Simmons et al., 2011) with the goal of having at least 100 participants in each condition. After providing informed consent and demographic background information, participants were randomly assigned to an experimental condition. The two written stimuli were derived from an adaptation of the transcript and 
Table 1 Results of conditional process analysis (moderated mediation model), Studies 1 and 2.

\begin{tabular}{|c|c|c|c|c|c|c|}
\hline & \multicolumn{2}{|c|}{ Emotional arousal } & \multicolumn{4}{|l|}{ Risk perception } \\
\hline & S1 & S2 & S1 & S2 & S2 & $\mathbf{S 2}$ \\
\hline Intercept & $3.52^{\star \star}(0.39)$ & $3.76^{\star \star}(0.27)$ & $6.18^{\star \star}(0.56)$ & $5.51^{\star \star}(0.56)$ & $7.38^{\star \star}(0.68)$ & $7.21^{\star \star}(0.37)$ \\
\hline $\begin{array}{l}\text { Affective ending (baseline: } \\
\text { positive) }\end{array}$ & $0.67^{\star \star}(0.22)$ & $0.46^{\star \star}(0.15)$ & $0.16(0.22)$ & $0.15(0.12)$ & $0.14(0.13)$ & $0.16(0.11)$ \\
\hline Emotional arousal $(M)$ & & & $0.13(0.10)$ & $0.25^{\star \star}(0.06)$ & $0.02(0.15)$ & $0.06(0.08)$ \\
\hline Moderate (W1) & & & $-2.64^{\star \star}(0.74)$ & $-1.51^{\star \star}(0.44)$ & & \\
\hline Conservative (W2) & & & $-3.93^{\star \star}(0.72)$ & $-3.35^{\star \star}(0.38)$ & & \\
\hline$M \times W 1$ & & & $0.34^{\star}(0.17)$ & $0.21^{\star}(0.09)$ & & \\
\hline$M \times W 2$ & & & $0.46^{\star \star}(0.17)$ & $0.42^{\star \star}(0.08)$ & & \\
\hline $\begin{array}{l}\text { Group (individualism vs. } \\
\text { communitarianism) }\end{array}$ & & & & & $-0.90^{\star \star}(0.17)$ & \\
\hline$M \times$ Group & & & & & $0.12^{\star \star}(0.04)$ & \\
\hline $\begin{array}{l}\text { Grid (hierarchy vs. } \\
\text { egalitarianism) }\end{array}$ & & & & & & $-1.05^{\star \star}(0.10)$ \\
\hline$M \times$ Grid & & & & & & $0.12^{\star \star}(0.02)$ \\
\hline Age & $0.00(0.01)$ & $0.01(0.01)$ & $-0.01(0.01)$ & $-0.02^{\star \star}(0.01)$ & $-0.02^{\star \star}(0.01)$ & $-0.02^{\star \star}(0.01)$ \\
\hline Gender & $0.37(0.23)$ & $0.08(0.16)$ & $0.14(0.23)$ & $0.22(0.12)$ & $0.27^{\star}(0.13)$ & $0.10(0.12)$ \\
\hline$R^{2}$ & 0.06 & 0.02 & 0.40 & 0.49 & 0.38 & 0.54 \\
\hline \multicolumn{7}{|l|}{ Index of moderated mediation } \\
\hline W1 & & & $0.23(-0.06,0.57)$ & $0.10(0.00,0.24)$ & & \\
\hline W2 & & & $0.31(0.01,0.77)$ & $0.19(0.05,0.37)$ & & \\
\hline Group & & & & & $0.06(0.01,0.12)$ & \\
\hline Grid & & & & & & $0.05(0.02,0.10)$ \\
\hline
\end{tabular}

photos of a climate change video, differing only in the valence of their endings (see Supplementary Information, Appendix A).

Qualified participants were asked to assess climate change risk using the item, "How much risk do you believe global warming poses to human health, safety, or prosperity?" (Kahan, 2017) on an 8-point Likert scale with anchors from $0=$ "none at all" to $7=$ "extremely high risk" $(M=5.42, \quad \mathrm{SD}=1.89)$. To gauge emotional arousal, we adapted a measure from Salgado and Kingo (2017) using the item, "How emotionally intense was it for you to read this article?", on a 7-point scale with anchors from $1=$ "minimal emotional intensity" to $7=$ "maximal emotional intensity" $(M=4.10, \mathrm{SD}=1.58)$. In a manipulation check, participants were asked to rate whether the appeal ended on a positive or negative note (optimistic: $M=4.63, \mathrm{SD}=1.49$; pessimistic: $M=1.92, \quad \mathrm{SD}=1.04 ; \quad t(198)=14.94, \quad p<0.000)$. Finally, participant political ideology was assessed (47.0\% liberals, $30.5 \%$ moderates, $22.5 \%$ conservatives), as well as additional items outside of the focus of this study.

Results. Consistent with our expectations, participants in the pessimistic condition reported greater risk perception $(M=5.66$, $\mathrm{SD}=1.79)$ than those in the optimistic condition $(M=5.20$, $\mathrm{SE}=1.97 ; \quad$ Mann-Whitney $U=4244, \quad p=0.055)$. Moreover, participants in the pessimistic condition reported higher emotional arousal $(M=4.44, \mathrm{SD}=1.57)$ than those in the optimistic condition $(M=3.75, \mathrm{SD}=1.53 ; t(198)=-3.17, p=0.002)$. A linear regression modelling risk perception further revealed a significant positive relationship with emotional arousal $(\beta=0.48$, $\mathrm{SE}=0.08, p<0.001, R^{2}=0.16$ ). These findings suggest a possible mediating role of emotional arousal on the relationship between affective ending and risk perception, a proposition we tested with conditional process analysis using the PROCESS 3.1 macro for SPSS (Hayes, 2013), controlling for age and gender. The analysis (Model 4; 10,000 bootstrap samples) revealed a significant indirect effect $(a b=0.31, \mathrm{SE}=0.12 ; 95 \% \mathrm{CI}: 0.10,0.56)$. Finally, we tested the moderating effect of political ideology, following a similar modeling approach (Model 14; 10,000 bootstrap samples; Table 1). The moderated mediation index for political moderates was not significant $(a b=0.23, \mathrm{SE}=0.16 ; 95 \% \mathrm{CI}:-0.06,0.57)$, whereas the moderated mediation index for conservatives was significant $(a b=0.31, \mathrm{SE}=0.20 ; 95 \% \mathrm{CI}: 0.01,0.77)$. An examination of the conditional indirect effects at different levels of the moderator (Fig. 1a) revealed that at lower levels of emotional arousal, risk perception is significantly lower for participants with conservative political ideology.

\section{Study 2: The influence of affective ending (optimistic vs. pessimistic) on emotional arousal and perception of climate change risk-video stimuli}

Experimental design. In a single factor (affective ending: optimistic $(N=228)$ vs. pessimistic $(N=221))$ between-subjects online experiment, 449 U.S.-based residents were recruited through Mturk $\left(49.9 \%\right.$ females, $\left.M_{\text {age }}=39.8, \mathrm{SD}_{\text {age }}=12.3\right)$ and compensated with USD 1.20. Sample size was determined beforehand with the goal of increasing the likelihood of recruiting participants with ideologically diverse political and cultural worldviews. The protocol was similar to Study 1 with the following exceptions: (i) stimuli included the actual video from which transcripts were adapted for study 1; (ii) the two video stimuli were identical except for the final $6 \mathrm{~s}$ that were professionally edited: the original video ended with positive valence, and the adapted version ended with negative valence; and (iii) after viewing the video, participants were asked to read the final two written appeal segments used in Study 1.

The statement assessing emotional arousal was adapted to the medium, and read, "how emotionally intense was it for you to watch this video?" Three measures of message receiver values were employed: (a) political ideology (39.9\% liberals, $25.4 \%$ moderates, $34.7 \%$ conservatives); (b) group worldviews (individualism vs. communitarianism; $M=3.68, \mathrm{SD}=1.05$ ); and (c) grid worldviews (hierarchy vs. egalitarianism; $M=2.78, \mathrm{SD}=1.40$ ). The two dimensions of cultural worldviews were assessed using the short-form of the cultural worldview scale (Kahan et al., 2010) (see Supplementary Information, Appendix B). Finally, in a manipulation check, participants were asked to rate whether the appeal ended on a positive or negative note (optimistic: $M=4.84$, 

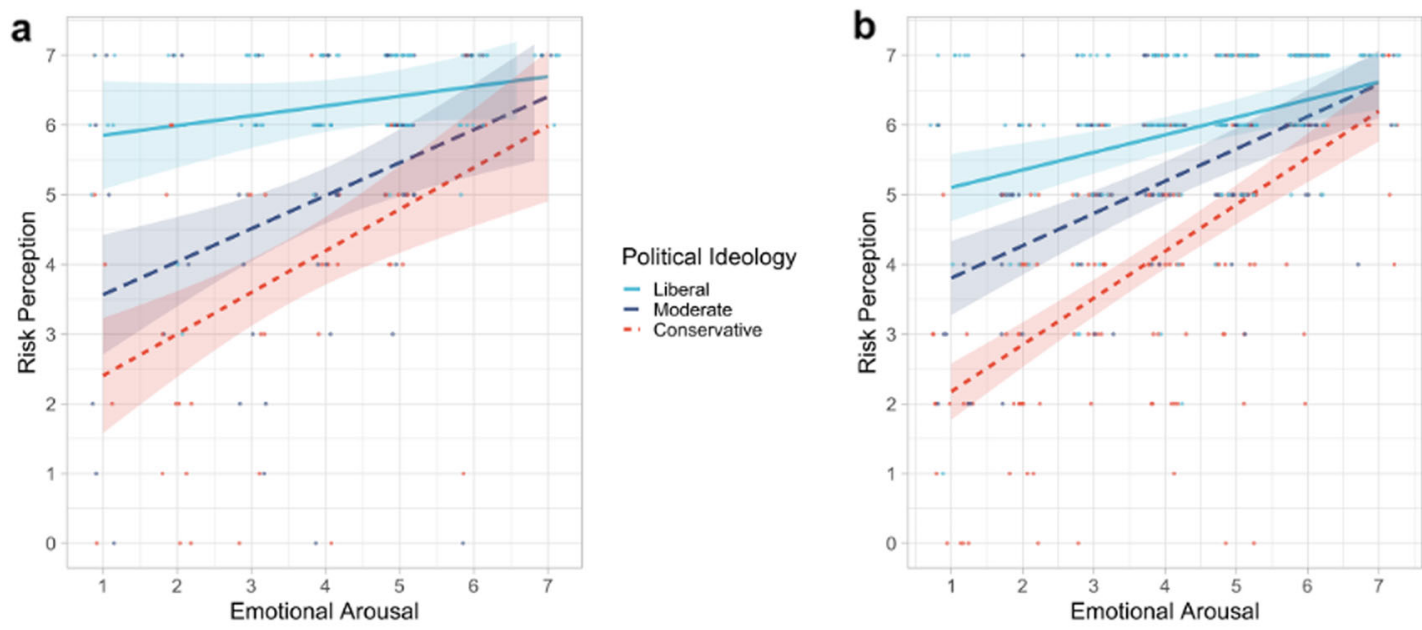

Political Ideology

- Liberal

- Moderate

-. Moderate
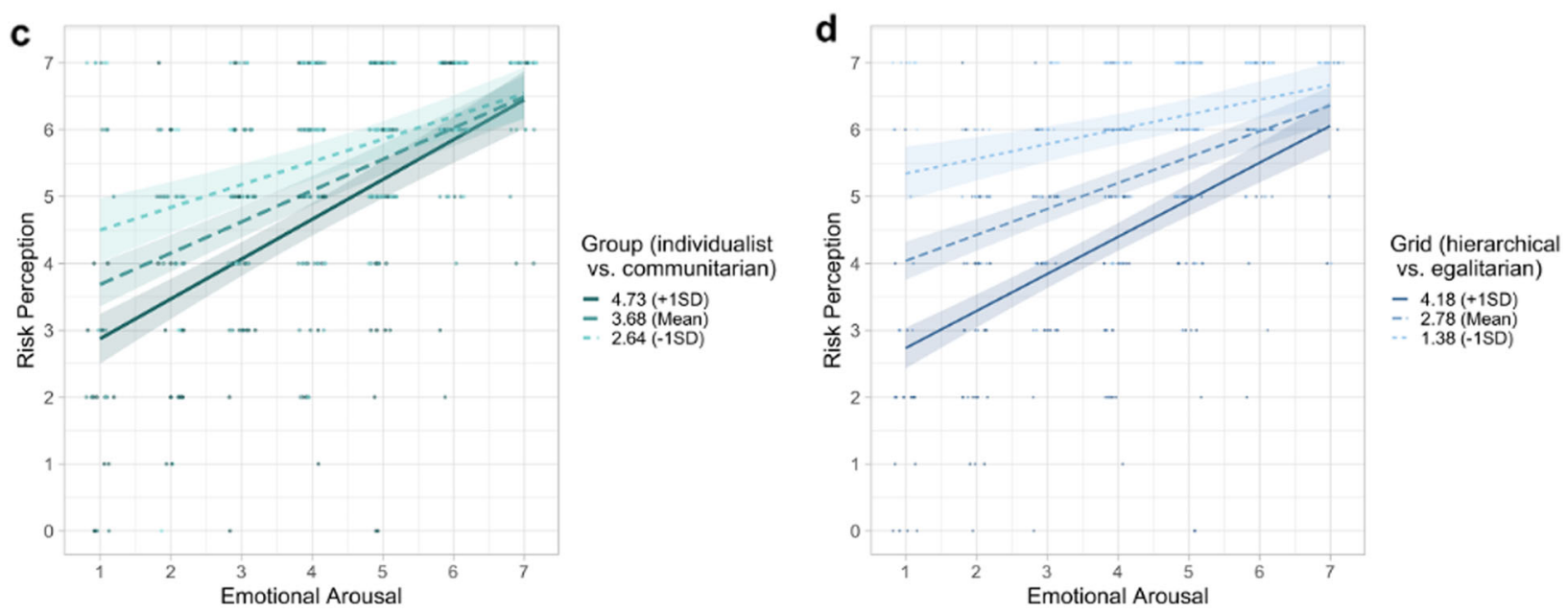

Fig. 1 Interaction effects. Graph (a): Study 1, interaction between risk perception and emotional arousal across moderator levels (political ideology: liberal, moderate, conservative). Graph (b): Study 2, interaction between risk perception and emotional arousal across moderator levels (political ideology: liberal, moderate, conservative). Graph (c): Study 3, interaction between risk perception and emotional arousal across moderator levels (Group cultural worldviews: individualists, communitarians). Graph (d): Study 3, interaction between risk perception and emotional arousal across moderator (Grid cultural worldviews: hierarchical, egalitarian).

$\mathrm{SD}=1.41$; pessimistic: $M=2.53, \mathrm{SD}=1.33, t(447)=17.85, p<$ $0.000)$.

Results. Consistent with our expectations, participants in the pessimistic condition reported greater risk perception $(M=5.56, \mathrm{SD}=1.53)$ than those in the optimistic condition $(M=5.17, \mathrm{SD}=1.83$; Mann-Whitney $U=22329, p=0.032)$. More, participants in the pessimistic condition reported higher emotional arousal $(M=4.49, \mathrm{SD}=1.57)$ than those in the optimistic condition $(M=4.02, \mathrm{SD}=1.67 ; t(447)=-3.08$, $p=0.002)$. A linear regression modelling risk perception further revealed a significant positive relationship with emotional arousal $\left(\beta=0.54, \mathrm{SE}=0.04, p<0.001 ; R^{2}\right.$ of 0.27$)$. Following an analytical approach similar to Study 1 , we tested the mediating effect of emotional arousal. The analysis revealed a significant indirect effect $(a b=0.25, \mathrm{SE}=0.08 ; \mathrm{CI}=0.09,0.41)$. Finally, we tested the moderating effects of political ideology, group, and grid cultural worldviews. All moderated mediation indices were significant (Table 1). An examination of the conditional indirect effects at different levels of the moderators (Fig. 1b-d) revealed that at lower levels of emotional arousal, risk perception is significantly lower for moderates and conservatives, as well as for lower levels of group and grid values (i.e., communitarians and egalitarians, respectively).

Discussion: Studies 1 and 2. The results of the first two studies provide support for our proposition that climate change appeals with a pessimistic ending trigger emotional arousal, which in turn influences perceptions of climate change risk. Moreover, the influence of emotional arousal on risk perception differs across people with varying political ideologies and cultural worldviews. The greatest differences are observed among conservatives and people holding individualist or hierarchical worldviews, who report higher levels of concern about climate change when presented with a pessimistic climate change appeal.

Study 3: the influence of affective ending (optimistic vs. pessimistic vs. fatalistic) on emotional arousal, climate change risk perception, and outcome efficacy

Experimental design. In Studies 1 and 2, we tested the two poles of affective ending (optimistic vs. pessimistic). Prior work suggests that people are more receptive to information when both threat appraisal and outcome efficacy are high (Witte and Allen, 2000). People might be scared into perceiving risk, but 
Table 2 Results of conditional process analysis (moderated mediation model), Study 3.

\begin{tabular}{|c|c|c|c|c|}
\hline & Emotional Arousal & Outcome efficacy & Outcome efficacy & Outcome efficacy \\
\hline Intercept & $4.12^{\star \star}(0.41)$ & $3.01^{\star \star}(0.20)$ & $4.51^{\star \star}(0.24)$ & $3.49^{\star \star}(0.19)$ \\
\hline Pessimistic (X1) & $0.41^{\star \star}(0.14)$ & $-0.06(0.06)$ & $-0.08(0.06)$ & $-0.04(0.06)$ \\
\hline Fatalistic (X2) & $0.50^{\star \star}(0.14)$ & $-0.02(0.06)$ & $-0.04(0.06)$ & $0.02(0.06)$ \\
\hline Conservative (W2) & & $-1.70^{\star \star}(0.14)$ & & \\
\hline$M \times W 1$ & & $0.14^{\star \star}(0.03)$ & & \\
\hline$M \times W 2$ & & $0.24^{\star \star}(0.03)$ & & \\
\hline Generalized self-efficacy & $-0.42^{\star \star}(0.12)$ & $0.03(0.05)$ & $0.10(0.05)$ & $0.08(0.05)$ \\
\hline Age & $0.00(0.00)$ & $0.00(0.00)$ & $-0.00(0.00)$ & $0.00(0.00)$ \\
\hline Gender & $0.79^{\star \star}(0.11)$ & $0.13^{*}(0.05)$ & $0.11^{\star}(0.05)$ & $0.06(0.05)$ \\
\hline & 0.07 & 0.34 & 0.35 & 0.42 \\
\hline \multicolumn{5}{|l|}{ Index of moderated mediation } \\
\hline W1 (for X1) & & $0.06(0.02,0.10)$ & & \\
\hline Grid (for X1) & & & & $0.02(0.01,0.03)$ \\
\hline Grid (for X2) & & & & $0.02(0.01,0.04)$ \\
\hline
\end{tabular}

how does this influence the sense that their own actions matter for solving the challenge of climate change? Thus, in Study 3, we added a third condition to test the difference between negative affective valence arising from pessimistic messaging with the possibility of a hopeful outcome (pessimistic condition) versus fatalistic messaging suggesting that it is too late to turn things around (fatalistic condition). Moreover, we introduced perceived outcome efficacy as a dependent measure to test the proposition that both negative conditions would outperform positive valence for sparking emotional arousal, motivating a stronger sense of agency and influence on outcomes related to climate change. Finally, we used a nationally representative sample of the U.S. population to increase the external validity of our findings.

In a single factor, between-subjects online experiment, 1115 US-based residents $\left(54.3 \%\right.$ females; $M_{\text {age }}=49.8, \mathrm{SD}_{\text {age }}=16.8$ ) were recruited through a nationally representative panel. Sample size was determined beforehand. The protocol and measures were similar to the two previous studies with the following exceptions: (i) in addition to optimistic $(N=375)$ and pessimistic $(N=375)$ conditions, a fatalistic condition was added $(N=365)$ (Supplementary Information, Appendix C); (ii) the main dependent variable, outcome efficacy, was operationalized using a single item, "I believe my actions have an influence on climate change" adapted from Kellstedt et al. (2008), and measured on a 4 -point scale with anchors $1=$ "strongly disagree" to $4=$ "strongly agree") $(M=2.91, \mathrm{SD}=0.99)$; and (c) the Generalized Self-Efficacy Scale was included to measure trait efficacy ${ }^{35}$ consisting of 10 items measured on a 4-point scale with anchors $1=$ "not at all true" to $4=$ "exactly true") $(M=3.18$, $\mathrm{SD}=0.48 ; \alpha=0.90)$. The same message receiver values were employed as in Study 2: (a) political ideology (31.6\% liberals, $35.2 \%$ moderates, $33.3 \%$ conservatives) (b) group cultural worldviews (individualism vs. communitarianism; $M=4.05$, $\mathrm{SD}=1.14$ ), and (c) grid cultural worldviews (hierarchy vs. egalitarianism; $M=3.05, \mathrm{SD}=1.60)$. Finally, in a manipulation check, participants were asked to rate whether the appeal ended on a positive or negative note $(F(2,1112)=70.63, p<0.001)$. Post hoc comparisons using Tukey's HSD test revealed that the optimistic condition was rated as more positive $(M=4.52$, $\mathrm{SD}=1.66)$ than the pessimistic ending condition $(M=3.36$, $\mathrm{SD}=1.92, p<0.001)$, with the latter being more positive than the fatalistic condition $(M=2.98, \mathrm{SD}=1.93, p=0.014)$.

Results. We did not observe any significant difference in outcome efficacy across the three conditions $(F(2,1112)=0.27, p=0.761)$, however, we did find significant differences in emotional arousal, $(F(2,1112)=8.34, p<0.001)$. Post hoc comparisons using Tukey's HSD test revealed that participants in the pessimistic condition reported higher emotional arousal $(M=3.69, \mathrm{SD}=1.84)$ than those in the optimistic condition $(M=3.22, \mathrm{SD}=1.89 ; p<0.001)$. However, there was no significant difference between the pessimistic and fatalistic conditions $(M=3.74, \mathrm{SD}=1.99 ; p=0.920)$. A linear regression with outcome efficacy as the dependent variable further revealed a significant positive association with emotional arousal $\left(\beta=0.22, \mathrm{SE}=0.01, p<0.001 ; R^{2}\right.$ of 0.18$)$.

Next, we tested the mediating effect of emotional arousal on the relationship between affective ending and outcome efficacy, using conditional process analysis controlling for age, gender, and trait efficacy. Analysis revealed significant indirect effects for the pessimistic $(a b=0.09, \mathrm{SE}=0.03 ; 95 \% \mathrm{CI}: 0.03,0.15)$, and fatalistic conditions $(a b=0.10, \mathrm{SE}=0.03 ; 95 \% \mathrm{CI}$ : $0.05,0.17)$. We then tested the moderating role of core values, operationalized as political ideology, group-, and grid cultural worldviews. All tested moderated mediation indices were significant (Table 2). An examination of the conditional indirect effects at different levels of the moderators (Fig. 2) revealed that perceived outcome efficacy is lower at lower levels of emotional arousal for all groups, but the effect is particularly pronounced for moderates and conservatives, as well as for those with lower levels of group and grid values (i.e., communitarians and egalitarians).

Discussion: Study 3. The results of Study 3 successfully replicate the findings of the two previous studies with a different 


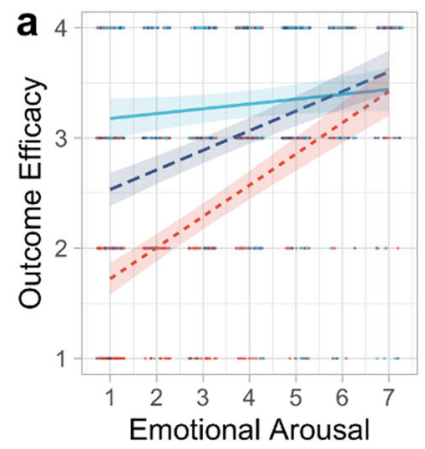

\section{Political Ideology \\ - Liberal \\ - Moderate \\ - Conservative}

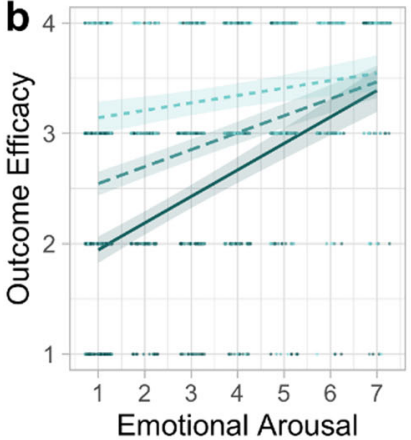

Group (individualist

vs. communitarian)

$\begin{array}{ll}- & 4.65(+1 \mathrm{SD}) \\ - & 3.05 \text { (Mean) }\end{array}$

-. $1.44(-1 \mathrm{SD})$

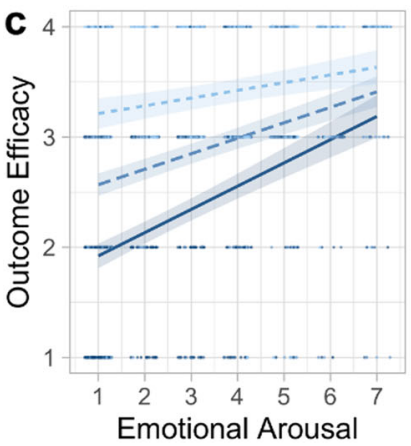

\section{Grid (hierarchist} vs. egalitarian)

$-4.65(+1 S D)$

- 3.05 (Mean)

$1.44(-1 \mathrm{SD})$

Fig. 2 Interaction effects between risk perception and emotional arousal across groups of moderators.

dependent variable: outcome efficacy. As in Studies 1 and 2, the difference in outcome efficacy as a result of increased emotional arousal was significantly greater in groups most likely to be disengaged with the issue, namely moderates and conservatives, and those holding communitarian and egalitarian cultural worldviews (Figs. 2 and 3).

\section{General discussion}

Our findings suggest that the affective ending of climate change appeals influences engagement. Pessimistic endings yield higher risk perception via heightened emotional arousal, and though this held true for all audiences, the effects are particularly pronounced in individuals usually exhibiting lowlevels of concern about climate change (i.e., moderates, conservatives, and those holding individualistic or hierarchical worldviews). Optimistic endings may comfort a public suffering from apocalypse fatigue, but do not appear to increase risk perception and perceived outcome efficacy. These findings are in line with recent experimental work finding that cardiac activity indicative of emotional arousal predicts behavioral engagement with climate change (Morris et al., 2019). In contrast to work positing that "fear appeals" may hinder efficacy (O’Neill and Nicholson-Cole, 2009), our results also suggest that pessimistic endings actually increase people's belief that their own individual behavior matters for climate change, even in the face of fatalistic messaging, though there are differences across ideologically diverse groups.

Research from the fields of neuroscience and social psychology offers plausible explanations for why conservatives might be more threat-reactive in the face of emotional arousal than liberals. While the formation and processing of emotion involve complex neural connectivity, there is evidence for the lateralization (asymmetrical representation) of brain function Lane and Nadel (2002). Liberalism has been associated with increased grey matter volume in the anterior cingulate cortex, an area of the brain associated with emotion regulation and executive function, which allows for greater cognitive flexibility (Kanai et al., 2011). Conservativism, on the other hand, has been associated with increased volume in the right hemisphere of the amygdala (Amodio et al., 2007; Kanai et al., 2011), an area which exerts greater influence on the processing of primary emotions such as fear, as well as emotional expression than the left hemisphere. These differences in brain functions/anatomy may be mappable to basic motivations for safety and survival and explain the association between political ideology and threat reactivity (Lilienfeld and Latzman, 2014; Pedersen et al., 2018). Napier et al. (2018) found, for example, that Republicans took significantly more liberal positions on social issues after envisioning feeling completely and totally physically safe, while Nail et al. (2009) found that dispositional liberals took on considerably more conservative positions in the face of systeminjustice and mortality salience threats.

These findings affirm the critical role of negative affect as a powerful lever for heightening perception of climate change risk but require further investigation. Climate change scholars, communicators, and policymakers should test segmentation strategies to assess the optimal degree of negativity in messaging designed for ideologically-and culturally diverse audiences. Given that public engagement with climate change is low in the United States (Leiserowitz et al., 2017), and even in Europe, very few people feel a sense of personal responsibility for its effects (European Commission, 2014), one possible remedy could be to tailor the affective ending of climate change appeals.

What are the long-term effects of various affective combinations on risk perception and efficacy, and do these effects persist over time? Do these vary across cultures? At what point might the use of negative affect backfire as a result of desensitization? These are important questions worth exploring in future work.

\section{Ethics statement}

The work was carried out in accordance with the Helsinki Declaration, taking every precaution to protect the privacy and interests of human participants, all of whom gave written, informed consent.

\section{Data availability}

The datasets for the three studies analyzed in this manuscript have been registered with the Open Science Framework. Complete stimulus materials are available in the Supplementary Information, and data can be accessed with permission from Open Science Framework: https://osf.io/bvqat/?view_only= 455db4ac6b744393920a1e056ddac8d1.

Received: 15 December 2019; Accepted: 12 August 2020;

Published online: 03 September 2020 


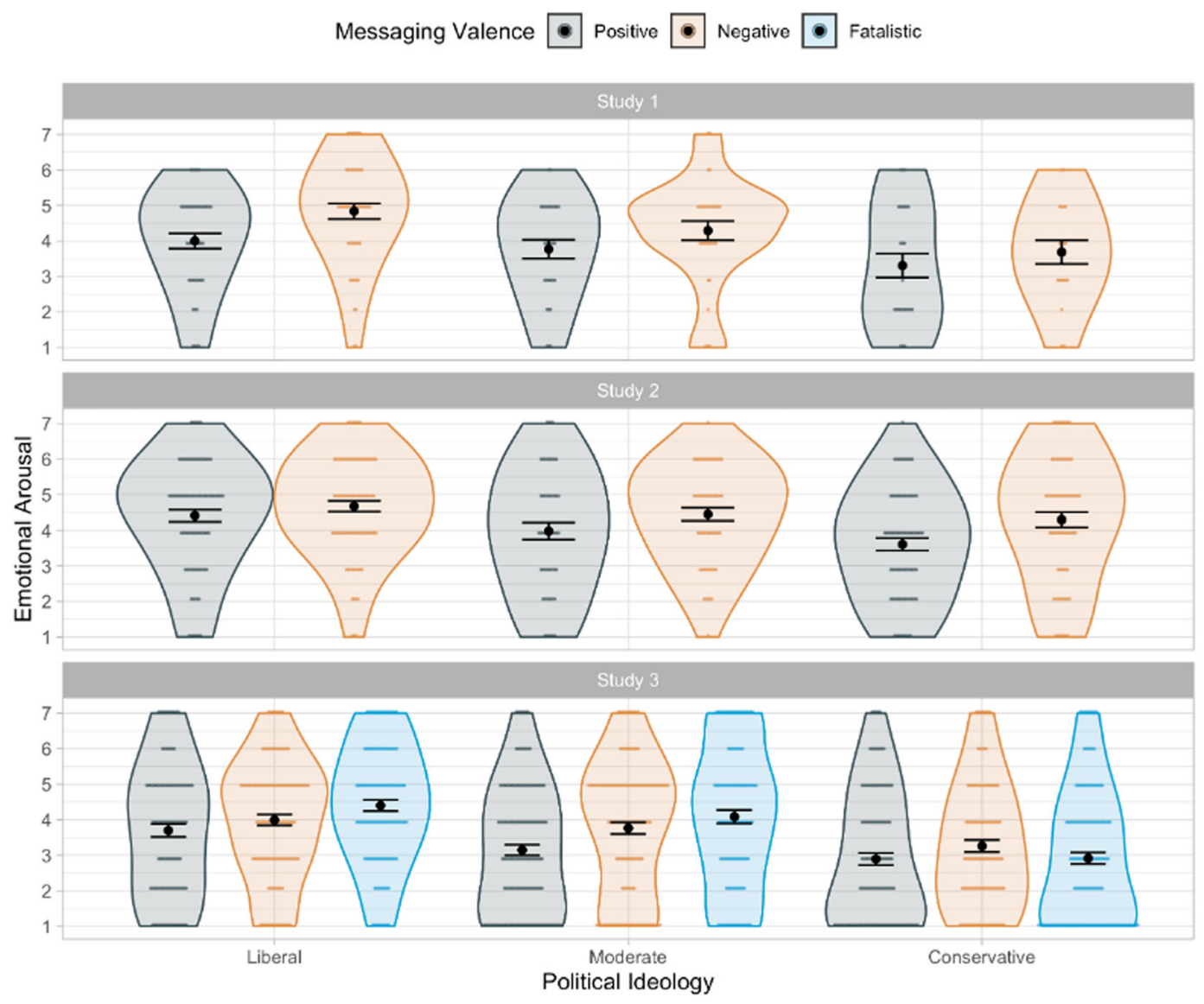

Fig. 3 Violin plots of experienced emotional arousal by condition and political ideology, Studies 1-3. Black dots represent means; error bars indicate standard errors.

\section{References}

Amodio DM, Jost JT, Master SL, Yee CM (2007) Neurocognitive correlates of liberalism and conservatism. Nat Neurosci 10(10):1246-1247

Bandura A (2002) Social foundations of thought and action. In D. F. Marks (Ed.), The health psychology reader (pp. 94-106). SAGE Publications Ltd, London. https://doi.org/10.4135/9781446221129.n6

Do AM, Rupert AV, Wolford G (2008) Evaluations of pleasurable experiences: the Peak-End Rule. Psychon Bull Rev 15(1):96-98

European Commission (2014) Climate change, Special barometer. European Commission. http://ec.europa.eu/public_opinion/archives/ebs/ebs_409_en.pdf

Feldman L, Hart PS (2015) Using political efficacy messages to increase climate activism: the mediating role of emotions. Sci Commun 38(1):99-127. https:// doi.org/10.1177/1075547015617941

Finucane ML, Alhakami A, Slovic P, Johnson SM (2000) The affect heuristic in judgments of risks and benefits. J Behav Decis Mak 13(1):1-17

Freedman A (2017) No, New York Mag: climate change won't make the earth uninhabitable by 2100. Mashable. https://mashable.com/2017/07/10/new-york-magclimate-story-inaccurate-doomsday-scenario/?europe $=$ true \#Qr0U8y0_wPqE

Ganzach Y (2000) Judging risk and return of financial assets. Organ Behav Hum Decision Process 83(2):353-370

Hayes AF (2013) Introduction to mediation, moderation, and conditional process analysis: a regression-based approach. Guilford Press, New York

Kahan DM (2015) Climate-science communication and the measurement problem. Political Psychol 36(S1):1-43. https://doi.org/10.1111/pops.12244

Kahan DM (2017) 'Ordinary science intelligence': a science-comprehension measure for study of risk and science communication, with notes on evolution and climate change. J Risk Res 20(8):995-1016. https://doi.org/10.1080/ 13669877.2016.1148067

Kahan DM, Braman D (2006) Cultural cognition and public policy. Yale Law Policy Rev 24(1):149-172

Kahan DM, Braman D, Cohen GL, Gastil J, Slovic P (2010) Who fears the hpv vaccine, who doesn't, and why? An experimental study of the mechanisms of cultural cognition. Law Hum Behav 34(6):501-516

Kahan DM, Braman D, Slovic P, Gastil J, Cohen G (2009) Cultural cognition of the risks and benefits of nanotechnology. Nat Nanotechnol 4(2):87
Kahan DM, Jenkins-Smith H, Braman D (2011) Cultural cognition of scientific consensus. J Risk Res 14(2):147-174. https://doi.org/10.1080/13669877.2010.511246

Kanai R, Feilden T, Firth C, Rees G (2011) Political orientations are correlated with brain structure in young adults. Current biology 21(8):677-680

Kellstedt PM, Zahran S, Vedlitz A (2008) Personal efficacy, the information environment, and attitudes toward global warming and climate change in the United States. Risk Anal 28(1):113-126

Lane RD, Nadel L (2002) Cognitive neuroscience of emotion. Oxford University Press

Leiserowitz A, Maibach E, Roser-Renouf C, Rosenthal S, Cutler M, Kotcher J (2017) Climate change in the American Mind: October 2017. Yale program on climate change communication. Yale University and George Mason University, New Haven, CT

Lilienfeld SO, Latzman RD (2014) Threat bias, not negativity bias, underpins differences in political ideology. Behav Brain Sci 37(3):318-319

Lorenzoni I, Nicholson-Cole S, Whitmarsh L (2007) Barriers perceived to engaging with climate change among the UK public and their policy implications. Glob Environ Change 17(3-4):445-459. https://doi.org/10.1016/j.gloenvcha.2007.01.004

Mann ME, Hassol SJ, Toles T (2017) Doomsday scenarios are as harmful as climate change denial. Washington Post. Retrieved from https://www.washingtonpost. com/opinions/doomsday-scenarios-are-as-harmful-as-climate-change-denial/ 2017/07/12/880ed002-6714-11e7-ald7-9a32c91c6f40_story.html

Mayer A, Smith EK (2019) Unstoppable climate change? The influence of fatalistic beliefs about climate change on behavioural change and willingness to pay cross-nationally. Clim Policy 19(4):511-523

McCright AM, Dunlap RE, Marquart-Pyatt ST (2016) Political ideology and views about climate change in the European Union. Environ Politics 25(2):338-358. https://doi.org/10.1080/09644016.2015.1090371

Morris BS, Chrysochou P, Christensen J, Orquin J, Barraza JA, Zak PJ, Mitkidis P (2019) Stories vs. facts: triggering visceral response to climate change. Clim Change

Nail PR, McGregor I, Drinkwater AE, Steele GM, Thompson AW (2009) Threat causes liberals to think like conservatives. J Exp Soc Psychol 45(4):901-907

Napier JL, Huang J, Vonasch AJ, Bargh JA (2018) Superheroes for change: physical safety promotes socially (but not economically) progressive attitudes among conservatives. Eur J Soc Psychol 48(2):187-195 
Nordhaus T, Shellenberger M (2009) Apocalypse fatigue: losing the public on climate change. Yale Environ 360:16

O’Neill S, Nicholson-Cole S (2009) "Fear won't do it" promoting positive engagement with climate change through visual and iconic representations. Sci Commun 30(3):355-379

Pedersen WS, Muftuler LT, Larson CL (2018) Conservatism and the neural circuitry of threat: economic conservatism predicts Greater Amygdala-Bnst connectivity during periods of threat vs. safety. Soc Cogn Affect Neurosci 13 (1):43-51

Peters E, Slovic P (2000) The springs of action: affective and analytical information processing in choice. Personal Soc Psychol Bull 26(12):1465-1475. https:// doi.org/10.1177/01461672002612002

Rottenstreich Y, Hsee CK (2001) Money, kisses, and electric shocks: on the affective psychology of risk. Psychol Sci 12(3):185-190

Salgado S, Kingo OS (2019) How is physiological arousal related to self-reported measures of emotional intensity and valence of events and their autobiographical memories? Conscious Cogn 75:102811. https://doi.org/10.1016/ j.concog.2019.102811

Schwartz D, Loewenstein G (2017) The chill of the moment: emotions and proenvironmental behavior. J Public Policy Market 36(2):255-268

Schwarzer R, Jerusalem M (1995) Generalized self-efficacy scale measures in health psychology: a user's portfolio. causal and control beliefs. NFER-Nelsen, Windsor, pp. 35-37

Sharot T (2011) The optimism bias. Curr Biol 21(23):R941-R945

Simmons JP, Nelson LD, Simonsohn U (2011) False-Positive Psychology: Undisclosed Flexibility in Data Collection and Analysis Allows Presenting Anything as Significant. Psychol Sci 22(11):1359-1366. https://doi.org/10.1177/ 0956797611417632

Slovic P, Finucane ML, Peters E, MacGregor DG (2004) Risk as analysis and risk as feelings: some thoughts about affect, reason, risk, and rationality. Risk Anal 24(2):311-322. https://doi.org/10.1111/j.0272-4332.2004.00433.x

Smith N, Leiserowitz A (2014) The role of emotion in global warming policy support and opposition. Risk Anal 34(5):937-948. https://doi.org/10.1111/ risa. 12140

Tversky A, Kahneman D (1992) Advances in prospect theory: cumulative representation of uncertainty. J Risk Uncertain 5(4):297-323

Weber EU (2006) Experience-based and description-based perceptions of longterm risk: why global warming does not scare us (yet). Clim Change 77 (1):103-120

Witte K, Allen M (2000) A meta-analysis of fear appeals: implications for effective public health campaigns. Health Educ Behav 27(5):591-615. https://doi.org/ $10.1177 / 109019810002700506$

Yang ZJ, Kahlor L (2013) What, me worry? The role of affect in information seeking and avoidance. Sci Commun 35(2):189-212
Yang ZJ, McComas KA, Gay G, Leonard JP, Dannenberg AJ, Dillon H (2011) Information seeking related to clinical trial enrollment. Commun Res 38 (6):856-882

\section{Acknowledgements}

The authors wish to thank David Salvesen and the University of North Carolina-Chapel Hill for sharing so generously from NC Climate Stories, from which our experimental stimuli were created. Thanks also to Jacob D. Christensen and Jacob L. Orquin for their helpful comments throughout this work. We are also enormously grateful for the support this research has received in the form of seed funding from the Interacting Minds Centre, Aarhus University, as well as the Aarhus University Research Foundation.

\section{Competing interests}

The authors declare no competing interests.

\section{Additional information}

Supplementary information is available for this paper at https://doi.org/10.1057/s41599 020-00574-z.

Correspondence and requests for materials should be addressed to B.S.M.

Reprints and permission information is available at http://www.nature.com/reprints

Publisher's note Springer Nature remains neutral with regard to jurisdictional claims in published maps and institutional affiliations.

\begin{abstract}
cc (i) Open Access This article is licensed under a Creative Commons C. Attribution 4.0 International License, which permits use, sharing, adaptation, distribution and reproduction in any medium or format, as long as you give appropriate credit to the original author(s) and the source, provide a link to the Creative Commons license, and indicate if changes were made. The images or other third party material in this article are included in the article's Creative Commons license, unless indicated otherwise in a credit line to the material. If material is not included in the article's Creative Commons license and your intended use is not permitted by statutory regulation or exceeds the permitted use, you will need to obtain permission directly from the copyright holder. To view a copy of this license, visit http://creativecommons.org/ licenses/by/4.0/.
\end{abstract}

(C) The Author(s) 2020 